Slavica

bruxellensia

\section{Slavica bruxellensia}

Revue polyphonique de littérature, culture et histoire

slaves

$8 \mid 2012$

Migration(s) et Exil(s)

\title{
Exotisation vs européanité : la bulgarité vue de Bulgarie et des États-Unis
}

Fizika na tăgata (Physique de la tristesse, 2011) de Georgi Gospodinov et Na iztok ot zapada (À l'Est de l'Ouest, 2011) de Miroslav Penkov

\section{Marie Vrinat-Nikolov}

\section{QpenEdition}

\section{Journals}

\section{Édition électronique}

URL : http://journals.openedition.org/slavica/1200

DOI : $10.4000 /$ slavica. 1200

ISSN : 2034-6395

\section{Éditeur}

Université libre de Bruxelles - ULB

Référence électronique

Marie Vrinat-Nikolov, «Exotisation vs européanité : la bulgarité vue de Bulgarie et des États-Unis », Slavica bruxellensia [En ligne], 8 | 2012, mis en ligne le 15 juin 2012, consulté le 03 mai 2019. URL http://journals.openedition.org/slavica/1200 ; DOI : 10.4000/slavica.1200

Ce document a été généré automatiquement le 3 mai 2019.

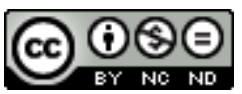

Les contenus de Slavica bruxellensia sont mis à disposition selon les termes de la Licence Creative Commons Attribution - Pas d'Utilisation Commerciale - Pas de Modification 3.0 France. 


\section{Exotisation vs européanité : la bulgarité vue de Bulgarie et des États-Unis}

Fizika na tăgata (Physique de la tristesse, 2011) de Georgi Gospodinov et $\mathrm{Na}$ iztok ot zapada (À l'Est de l'Ouest, 2011) de Miroslav Penkov

\section{Marie Vrinat-Nikolov}

1 Dans Gorki talog iskustva (Le résidu amer de l'expérience, 1998), l'écrivain serbe Danilo Kiš (1935-1989) confie :

On continue d'écrire chez nous une mauvaise prose, anachronique dans l'expression et les thèmes, entièrement appuyée sur la tradition du XIX ${ }^{e}$ siècle, une prose timide dans l'expérimentation, régionale, locale, dans laquelle cette couleur locale n'est en fait le plus souvent qu'un moyen d'essayer de préserver l'identité nationale en tant qu'essence de la prose. (...). Quant au monde, il continue de rechercher plus ou moins dans nos littératures l'excès, la couleur locale ou le pamphlet politique, des substituts du tourisme et de la politique. ${ }^{1}$

Constat désabusé, qu'il convient d'ailleurs de replacer dans son historicité, mais qui vaut également en partie pour la littérature bulgare et, sans doute, pour une grande partie des littératures balkaniques (que l'on pense au Macédonien Luan Starova [né en 1941], au Bulgare Anton Dončev [né en 1930] ou à l'Albanais Ismail Kadare [né en 1936] et à leur succès, franc ou relatif, à l'Ouest), lieux de prédilection à la fois d'une exotisation, d'une couleur locale et d'une affirmation identitaire douteuses, susceptibles d'être instrumentalisées par les politiques et par les gouvernants les appliquant.

Il est intéressant d'observer, dans la littérature bulgare, un changement très net de paradigme depuis ce qu'on appelle communément « la génération des années 1990 » qui a expérimenté avec la langue, les formes et les thèmes, a voulu en finir avec la couleur locale et montrer à la face du monde que cette littérature faisait partie intégrante du patrimoine européen, voire mondial. Ce faisant, ses auteurs ont ravivé la sempiternelle tension nous/les autres, national/étranger, orientalité/balkanité/européanité, qui a fait 
l'objet de débats persistants dans la première décennie $\mathrm{du} \mathrm{xxI}^{\mathrm{e}}$ siècle, tout comme elle était au cœur de la querelle entre Jeunes et Anciens au tournant des $\mathrm{XIX}^{\mathrm{e}}$ et $\mathrm{XX}^{\mathrm{e}}$ siècles.

Depuis quelques années, on assiste à l'essor d'une écriture de la diaspora, soit en bulgare, soit dans la «langue de l'autre» et l'on peut se demander comment elle se situe par rapport à cette tension : l'ignore-t-elle au contact d'autres cultures ou, au contraire, la vit-elle plus intensément que les écrivains «de l'intérieur»? Et, question corollaire, peut-on parler d'une poétique différente?

5 C'est à ces questions que je me propose d'apporter des éléments de réponse en m'appuyant sur deux œuvres emblématiques, me semble-t-il, de la représentation de soi et des autres, le roman Fizika na tăgata (Physique de la tristesse, 2011) de Georgi Gospodinov (né en 1968), écrit en bulgare et en Bulgarie, et le recueil de nouvelles Na iztok ot zapada (À l'Est de l'Ouest) de Miroslav Penkov (né en 1982), établi aux États-Unis depuis 2001, qui a écrit les textes de ce livre d'abord en anglais (New York, 2011), puis en bulgare, sa langue maternelle (Sofia, 2011).

\section{La tension nous/les autres, bulgarité/européanité au $\mathrm{xxI}{ }^{\mathrm{e}}$ siècle}

6 Du Réveil national (1762-1878), période d'avant la libération de la domination ottomane, pré-étatique, la littérature bulgare a hérité un rapport ambigu à l'Autre : d'une part, la majorité des hommes de lettres acteurs du Réveil national ont un accès enrichissant et formateur aux littératures étrangères, soit directement, soit par la traduction, qu'ils traduisent pour un grand nombre d'entre eux (c'est d'ailleurs en grande partie grâce à « l'épreuve de l'étranger » que la littérature bulgare se dote des genres modernes, prose, poésie et dramaturgie) ; d'autre part, l'arrivée massive et nouvelle d'œuvres étrangères classiques ou populaires traduites en bulgare effraie: ne vont-elles pas étouffer la littérature nationale (re)naissante? Cette dernière ne risque-t-elle pas de perdre son originalité?

7 Cette tension est sous-jacente, au tournant $\mathrm{du} \mathrm{xx}^{\mathrm{e}}$ siècle, dans le conflit entre Jeunes et Anciens qui opposa le cercle moderniste Misăl, plus particulièrement le poète et critique Penčo Slavejkov (1866-1912) au "Patriarche» de la littérature bulgare, Ivan Vazov (1850-1921), conflit demeuré célèbre dans l'histoire de la littérature bulgare, symbolisé par la fameuse étude du docteur Krăstjo Krăstev intitulée Mladi i stari, kritičeski očerki vărhu dnešnata bălgarska literatura (Jeunes et Anciens, esquisses littéraires sur la littérature bulgare d'aujourd'hui). Prônant l'émergence d'une littérature en soi et pour soi, la liberté du créateur, voulant concilier l'étranger et le national au nom de l'universel et dépasser les tendances collectives et populistes repliées sur la terre natale, Slavejkov fustige le caractère conjoncturel de l'œuvre de ses prédécesseurs ou contemporains repliés sur le passé ou happés par la bêtise environnante et s'afflige de l'état de la littérature : pour lui, il y a en Bulgarie des «lettres » - knižnina - mais pas encore de véritable littérature literatura. Toute l'œuvre de Slavejkov, aussi bien critique que poétique, vise à donner à la littérature bulgare de nouveaux concepts, des valeurs spirituelles jugées universelles pour la rapprocher des littératures européennes.

8 C'est encore cette tension qui est à l'œuvre durant tout le $\mathrm{xx}^{\mathrm{e}}$ siècle, dans ses deux extrêmes (européanisation de la littérature bulgare/repli sur la terre natale et sur soi) ou dans les tentatives faites pour les concilier. 
9 Au tournant du xxI ${ }^{e}$ siècle, le conflit Jeunes/Anciens est ravivé, au sortir de quarante-cinq ans de totalitarisme, mais retourné: le désir de traduction va dans le sens d'une exportation des valeurs littéraires nationales, et, cette fois, ce sont les « Anciens » qui accusent les «Jeunes » de céder à la tentation d'imiter les littératures ouest-européennes et américaine, dans le but non seulement de retrouver un public national qui s'en était détourné après les changements de 1989, mais aussi et surtout de gagner une présence dans la « République mondiale des Lettres».

10 Ces termes sont somme toute assez relatifs et ne recouvrent pas une différence générationnelle, mais politique et esthétique : d'un côté, la génération des années 1990 est celle qui a profondément renouvelé la littérature bulgare du postcommunisme, à la faveur de la liberté d'écrire retrouvée, par sa volonté de ne pas fixer ni formater la littérature qui prend des allures de « collectionneuse » de traditions diverses et par le jeu postmoderne avec les formes et avec les mots. C'est aussi une génération aux idées libérales, favorable à la collaboration avec les forces de l'OTAN et à l'adhésion à l'Union européenne. De l'autre, les « Anciens » sont plutôt proches du Parti socialiste bulgare issu de l'ancien parti communiste, ou des nationalistes, ils prônent une littérature qui ait « du sens » et ne cessent d'accuser les «Jeunes » d'écrire des textes vains, dénués de sens, de bafouer la sacro-sainte tradition, de ne pas respecter les valeurs traditionnelles bulgares, de vouloir imiter l'Occident sans avoir réellement de talent ni d'idées originales.

11 Ainsi, en janvier 2008, le critique et universitaire angliciste Vladimir Trendafilov relance la polémique dans l'hebdomadaire Kultura par un article intitulé «Krizata, kojato obnadeždava: nasledstvoto ot evropejzacijata » (La crise qui donne espoir : l'héritage de l'européanisation)2. Il s'agit de montrer que la jeune génération (sont nommés Gospodinov, Emilija Dvorjanova [née en 1958] et Teodora Dimova [née en 1960], des auteurs traduits à l'étranger où ils sont même primés) imite "les conceptions et les techniques du postmodernisme français ». Pour lui, le paradoxe, c'est que ces auteurs "s'auto-identifient » comme "premiers », «fondateurs », " originaux » sur la base, en fait, de « l'imitation de modèles étrangers ».

12 On peut donc parler de deux lignes traversant la littérature bulgare, en reprenant les termes de Kiš : une ligne exotisante, «timide dans l'expérimentation, régionale, locale » qui mise sur la couleur locale ; une ligne au contraire qui n'hésite pas à expérimenter, aussi bien dans les thèmes que dans la forme, qui rejette la couleur locale, l'exotisme, et se veut « universalisante ».

13 Elles sont représentées, de manière d'ailleurs paradoxale comme on le verra, par le roman Fizika na tăgata et par le recueil de nouvelles Na iztok ot zapada, tous les deux parus à la fin de l'année 2011.

\section{Na iztok ot zapada ou la bulgarité exotisée}

Ce recueil, d'abord écrit en anglais puis réécrit en bulgare par son auteur, porte un soustitre éloquent dans son édition anglaise de 2011 : A Country in stories ${ }^{3}$. Il s'agit de l'œuvre d'un jeune écrivain bulgare, qui signe là son premier livre. Arrivé aux États-Unis en 2001 pour y faire des études de psychologie, il enseigne actuellement l'écriture (creative writing ) à l'université du Nord-Texas. Son site web personnel ${ }^{4}$ fournit des informations très intéressantes qui éclairent la genèse de ce recueil. Dans une interview donnée à la télévision bulgare en décembre 2011, il confie avoir écrit des récits de science-fiction tant 
qu'il était en Bulgarie, des récits très " américanisés » selon son expression, inspirés de ses auteurs américains favoris. Mais aux États-Unis, il prend conscience que ce type d'écriture n'intéressera personne. Le hasard fait que son professeur d'histoire écrit un ouvrage sur les janissaires des Balkans. Apprenant que Penkov (né en 1982) est bulgare, il lui demande de lui résumer l'étude bien connue d'une historienne bulgare sur le sujet. C'est ainsi que le jeune homme se familiarise avec des pans de sa propre histoire qui ne lui étaient pas bien connus, celle des territoires bulgares sous la domination ottomane et du devşirme, appelé « impôt du sang » dans plusieurs langues balkaniques : il s'agit de la pratique selon laquelle de jeunes garçons chrétiens étaient enlevés, de gré ou de force, à leurs parents, islamisés et enrôlés dans l'armée du Sultan où il formaient un corps particulier.

Désireux de mettre à profit ses connaissances, impressionné par cette histoire qu'il met en parallèle, surtout lorsque cette pratique était consentie, voire demandée par des parents soucieux de l'avenir de leurs enfants, avec sa trajectoire personnelle et le fait que ses parents l'aient incité à tenter sa chance aux États-Unis, il l'insère dans son livre : l'une des nouvelles du recueil s'intitule précisément Devširme et mêle l'histoire personnelle d'un Bulgare émigré aux États-Unis et celle d'un janissaire qui finit par tomber amoureux d'une Bulgare, pour leur malheur, avec toutes les techniques narratives de l'histoirelégende: utilisation du médiatif, ce temps de l'histoire, de la légende et des contes, vocabulaire archaïsant, structures linguistiques populaires.

C'est donc la nostalgie pour son pays natal qui l'inspire et l'incite à vouloir faire connaître la Bulgarie à des lecteurs étrangers et à écrire son livre (on se rappelle que Pod igoto [Sous le joug $]^{5}, 1889$ ), LE grand roman national bulgare, épique et patriotique, fut écrit lorsque son auteur était en exil à Odessa et nostalgique de son pays). Toutes les circonstances entourant la genèse de ce livre éclairent un paradoxe: voici un auteur jeune, qui vit à l'étranger, au contact d'une littérature et d'une culture autres, et qui offre à son public une œuvre rappelant les nouvelles d'un Nikolaj Hajtov ${ }^{6}$ (1919-2002) ou les romans d'Ivajlo Petrov (1923-2005) ${ }^{7}$, par l'empathie non dépourvue d'humour à l'égard des Bulgares des villages, de leurs qualités comme de leurs travers, par la recherche de l'exotisation, de la couleur locale, voire d'une certaine linéarité, d'une facture « classique ».

Il en est ainsi dans la nouvelle « Na iztok ot zapada » qui donne son nom à tout le recueil, histoire d'un jeune garçon amoureux de sa cousine Vera qu'il ne voit qu'à l'occasion des kermesses de leurs villages respectifs, étant donné qu'ils sont séparés par le Danube et donc ressortissants de deux pays différents : le narrateur de la Bulgarie socialiste, Vera de la Yougoslavie où l'on trouve des jeans, des baskets et autres biens de consommation inexistants en Bulgarie. Tout comme sous la plume de Hajtov ou de Petrov, le registre est une imitation de la langue parlée, voire familière, des villages, le vocabulaire archaïsant, celui des chroniques («tărkulnaha se pet leta» [cinq années se sont écoulées], « dogdeto » [jusqu'à ce que], etc.). La scène théâtralisée (avec un humour bienveillant) montrant l'arrivée de celui qui fait passer d'une rive à l'autre est chargée d'un exotisme recherché :

Voici comment nous le traversons :

Rugissement au-dessus de l'eau et volutes de fumée. Mihalaki arrive dans sa barque. C'est une barque glorieuse. Pas une barque, mais un radeau à moteur. À l'une des extrémités, Mihalaki a fixé la banquette d'une vieille Moskvitch ${ }^{8}$, avant de la tapisser d'une peau de chèvre. Les poils à l'extérieur. Avec des taches noires et blanches et un peu de brun. Le voici, assis sur son trône : tranquille, terrifiant. Il 
mâchouille une pipe à tuyau d'ébène, tandis que ses longs cheveux blancs s'agitent derrière lui comme un étendard. ${ }^{9}$

Dans le droit fil d'une tradition narrative, l'histoire du village, celle de l'église ensevelie sous les eaux du Danube, sont racontées, deux types «d'idéal » s'affrontent, identité contre consommation :

Ses yeux s'assombrirent. Elle resta un long moment sans les ouvrir. Je compris. Elle était triste. Quant à moi, j'étais content. Elle avait des baskets flambant neuf et des jeans chers. Elle pouvait écouter des groupes occidentaux. Mais moi, j'avais quelque chose qu'on lui avait pris irrémédiablement.

— Je suis l'unique Bulgare ici, lui dis-je. ${ }^{10}$

La même empathie, non dépourvue d'humour, se lit à l'égard d'une certaine philosophie paysanne transmise de père en fils :

— J'ai mal aux mains, dis-je en pleurnichant. Le volant n'arrête pas de bouger.

- Nez-tordu ${ }^{11}$, me disait mon père, tu as assez gémi. Ce n'est pas un volant que tu tiens. C'est la vie que tu serres à la gorge. Serre, mon fils. Apprends à la serrer bien fort, cette vermine, parce que cette vermine, mon fils, il y a beau temps qu'elle sait te serrer. ${ }^{12}$

Revenons à la réflexion désabusée de Kiš : le monde, dit-il, « continue de rechercher plus ou moins dans nos littératures l'excès, la couleur locale ou le pamphlet politique ». La réception faite au livre de Penkov semble lui donner raison. À peine sorti aux États-Unis, où plusieurs critiques de grands journaux sont élogieux, il est déjà promis à treize éditions en langue étrangère ${ }^{13}$. Succès favorisé de surcroît par la présence d'un agent littéraire et par le fait que le livre a d'abord paru en anglais. Constatant que les éditions Héloïse d'Ormesson ont manifesté leur intention de publier ce recueil en français (à partir de l'original anglais), on peut se demander comment ce livre, entièrement centré sur une bulgarité traditionnelle, voire folklorisée, pourra être traduit par un traducteur de l'anglais ne connaissant rien à l'histoire, à la culture et aux traditions bulgares... Mais c'est une autre question.

Paradoxalement, c'est dans l'œuvre de Gospodinov, écrivain qui vit et crée en Bulgarie et en bulgare, que l'on trouve expérimentation, « européanisation », ainsi que la recherche d'une forme d'universalité.

\section{Fizika na tăgata : je sommes nous}

Fizika na tăgata, écrit en grande partie en Bulgarie, mais aussi à Berlin où son auteur a bénéficié d'un séjour d'un an par le biais du DAAD, est le second roman de Gospodinov après Estestven roman ${ }^{14}$ paru en 1999, traduit dans dix-huit langues. Ce premier roman avait catapulté son auteur sur le devant de la scène littéraire bulgare et a largement contribué à faire du roman le genre dominant dans la littérature bulgare du XxI ${ }^{\mathrm{e}}$ siècle (au lieu de la poésie). Sans compter un recueil de récits, deux pièces et deux recueils de poèmes, des chroniques parues dans le quotidien Dnevnik et son travail de rédacteur en chef de Literaturen vestnik (Journal littéraire), l'incubateur du post-modernisme et du renouvellement de la littérature en Bulgarie.

Fizika na tăgata, roman-labyrinthe,apparaît comme un prolongement/dépassement longuement et patiemment mûri de la quête du moi déjà à l'œuvre dans Un Roman naturel, qui englobe tous les autres «moi », et ce, dès le tout début du roman, dans son prologue où il déclare : 
Je suis né à la fin du mois d'août 1913, être humain de sexe masculin. Je ne sais pas la date exacte. On a attendu de voir quelques jours si j'allais survivre et c'est alors seulement qu'on m'a déclaré. (...) Je suis né deux heures avant le lever du soleil, mouche à vin. Je mourrai ce soir après le coucher du soleil. (...) Je suis né le $1^{\text {er }}$ janvier 1968, être humain de sexe masculin. Je me souviens dans le détail de toute l'année 1968, du début jusqu'à la fin. Je ne me rappelle rien de l'année en cours. Je ne sais même pas son numéro. (...) J'ai toujours été né. Je me rappelle encore le début de l'Ère de glace et la fin de la Guerre froide. Le spectacle de dinosaures mourants (durant ces deux époques) est l'une des choses les plus insoutenables que j'aie jamais vues. (...) Je ne suis pas encore né. Je suis à venir. J'ai moins sept mois. Je ne sais pas comment on compte ce temps négatif passé dans le ventre. (...) Je suis né le 6 septembre 1944, être humain de sexe masculin. Temps de guerre. Une semaine plus tard, mon père est parti sur le front. (...) J'ai des souvenirs de moi né comme buisson d'églantier, perdrix, ginkgo biloba, escargot, nuage de juin (ce souvenir est fugace), crocus mauve d'automne au bord du Halensee, cerisier précoce figé par une neige tardive d'avril, comme une neige ayant figé un cerisier leurré...

Je suis nous. ${ }^{15}$

Dans le labyrinthe des histoires, mais aussi l'originel, celui du Minotaure, alter ego du narrateur, Gospodinov va très loin dans cette démultiplication des je qui se révèle existentielle dans Fizika na tăgata :que de non-vécu, de manqué, de passé à côté, de laissé de côté dans une existence... "Si nous sommes tristes, ce n'est pas parce que nous sommes bulgares, mais parce que nous sommes des humains. La tristesse de Gospodinov est nostalgie de la complétude perdue : c'est le prix que nous avons payé pour devenir ce que nous sommes. $»^{16}$

tiples fils d'Ariane relient ce moi incomplet, hic et nunc, aux autres «moi » d'autres lieux et d'autres époques, humains, animaux ou plantes, le transformant en un moi collectif, empathique, plus exactement atteint du "syndrome empathico-somatique obsessionnel » qui lui permet de traverser les âges et d'entrer tour à tour dans les histoires et les corps de son grand-père, dans la Hongrie de 1945, du Minotaure, de Georgi Gospodinov, dans la Bulgarie communiste et post-communiste de 1968 à 2011, d'une mouche à vin, d'un nuage de printemps, d'une perdrix, etc. Avec l'enfance prend fin l'empathie. Le moi collectionne, « achète » alors les histoires d'autrui, encapsule le temps. Pour retarder la fin du monde. Pour ne pas oublier. Ce que l'on oublie habituellement, le périssable, l'éphémère, le quotidien, l'oublié par la "Grande Histoire ", le Minotaure. Parce que le sublime est partout, même dans «l'architecture, la physique et la métaphysique de la bouse de buffle ». Parce que le passé est le seul futur possible. Pour atteindre une «mémoire archétypale ». Pour que ceux qui naîtront après les apocalypses découvrent ce « kit de survie » et se souviennent de ce qui a été.

Cette quête de l'universel mise en œuvre dans Fizika na tăgata me paraît reposer précisément sur ce que le chercheur Ivan Mladenov définit comme "l'étrangéité mémorée » à propos de la littérature bulgare qu'il tente de conceptualiser dans son ouvrage Otklonena literatura (Une littérature déviée) ${ }^{17}$, et qui fonde, selon lui, l'universalité d'une littérature, d'une écriture: tout ce qu'une littérature nationale a accumulé au contact d'autres littératures, d'autres cultures, toute cette part d'étrangéité qu'elle garde en mémoire et qui lui confère son épaisseur et son universalité.

La mythologie, chez Gospodinov, les références à des personnages ou des lieux historiques, connus, font partie intégrante $\mathrm{du}$ « moi » qui les a incorporés et qui se dote ainsi d'une universalité. "Je sommes nous ». «Je » suis les autres. "Je » est citoyen du monde : 
Désormais, je sais. Je ne veux plus revivre aucun de ce qu'on appelle les événements de ma propre vie : ni ce premier événement de la naissance ni le dernier à venir, les deux étant tout autant inconfortables. (...) Rien n'est définitif, rien ne m'est encore arrivé. Tout le temps du monde est devant moi.

On retrouve dans ce roman, comme dans Estestven roman, la structure ouverte et collectionneuse, l'écriture fragmentée mêlant plusieurs types de narration et rattachant le « moi » non seulement à l'histoire d'un peuple, mais aussi à l'histoire mondiale. Car la peur obsessionnelle des narrateurs de Gospodinov n'est pas la fin du «moi » ni la fin de son pays natal, mais l'Apocalypse, la fin du monde. Cette architecture, qui veut épouser celle du labyrinthe, est soulignée par les sous-chapitres intitulés " couloirs latéraux » et par les arrêts :

Dans un article à paraittre, consacré à l'œuvre de trois écrivains d'origine bulgare vivant dans la diaspora, je conclue :

Ce qu'apportent à la littérature bulgare ces écrivains en marge du répertoire national parce qu'ils écrivent dans une autre langue, c'est, non pas une esthétique nouvelle (elle est profondément renouvelée en Bulgarie par certains écrivains contemporains), mais un regard plus distancié, notamment par rapport à tout ce qui constitue la mythologie identitaire nationale, et un appel à dépasser les frontières étroites du national pour accéder au regard du citoyen du monde.

Avec le recueil Na iztok ot zapada de Penkov, nous sommes devant un autre cas d'écriture dans la diaspora: la recherche des racines ne s'accompagne plus d'un regard distancié, mais d'une empathie à l'égard de tout ce qui constitue la couleur locale et d'un désir de faire découvrir le pays de l'enfance, certes l'un des plus mal connus de l'Union européenne. Ce qui suppose une écriture « exotisante » qui rappelle le ton des contes, des chroniques et des légendes traditionnelles. En revanche, nous retrouvons dans Fizika na tăgata de Gospodinov l'une des « obsessions » de son auteur : le « moi » individuel ne peut avoir de sens qu'en tant que maillon d'une grande chaîne collective qui le rattache à l'humanité tout entière. Pour ce faire, l'écriture ne saurait être linéaire, mais fragmentaire, labyrinthique, faite de prolepses et d'analepses, collectionneuse d'histoires et de «moi » divers. En même temps, elle répond au second volet du constat de Danilo Kiš :

(...) tandis que des thèmes et des moyens d'expression contemporains, l'esprit et le goût de l'expérimentation et de la nouveauté, la modernité, c'est ce qui est capable de créer un lien avec le lecteur d'aujourd'hui, quels que soient sa culture et son appartenance nationales ${ }^{19}$.

\section{NOTES}

1. Kiš D., Le résidu amer de l'expérience : entretiens, traduit du serbo-croate par Pascale Delpech, Fayard, Paris, 1998, p. 72 Version originale : Kiš D., Gorki talog iskustva, BIGZ, SKZ, Narodna Knjiga, Belgrade, 1990, $313 \mathrm{p}$.

2. Voir http://liternet.bg/publish8/vtrendafilov/krizata.htm. 
3. Penkov M., East of the West: A Country in stories (L'Est de l'Ouest : Un pays en histoires), Farrar, Straus and Giroux, New-York, 2011, 240 p.

4. http://www.miroslavpenkov.com/

5. Vazov I., Sous le joug, traduit du bulgare par Marie Vrinat, Fayard, Paris, 2007, 481 p. Il s'agit du premier roman en langue bulgare ; il fut publié en 1894 (NdlR).

6. Par exemple Divi razkazi (Histoires sauvages), Khr. G. Danov, Plovdiv, 1967, 193 p.

7. Par exemple Predi da se rodja i sled tova (Avant ma naissance et après, 1968) ou Hajka za vălci (trad, 1987) dans sa version intégrale. Le premier est disponible en français : Avant ma naissance et après, traduit par Marie Vrinat, Lausanne, L'Âge d'Homme, 1994, 181 p.

8. Voiture soviétique. (NdlA)

9. Penkov M., Na iztok ot zapada (À l'Est de l'Ouest), Ciela, Sofia, 2011, p. 31.

10. Ibid., p. 36

11. C'est le surnom du narrateur depuis qu'il s'est fait casser le nez par sa cousine. (NdlA)

12. Penkov M., Op. cit., p. 33.

13. Information disponible sur le site de l'auteur.

14. Paru en français : Un Roman naturel, traduit par Marie Vrinat, Phebus, Paris, 2002, 192 p.

15. Gospodinov G., Fizika na tăgata (Physique de la tristesse), Janet-45, Sofia, 2011, pp. 13-14.

16. Penčev B., «Gospodinov, tăgata i Minotavăra » (Gospodinov, la tristesse et le Minotaure), in : Kapital, 5 janvier 2012.

17. Mladenov I., Otklonena literatura (Une littérature déviée), Paradigma, Sofia, 2011, 246 p.

18. Gospodinov G., op. cit. p. 53.

19. Kiš D., op. cit., p. 72.

\section{INDEX}

Mots-clés : émigration, exil, littérature bulgare, réception, relation occident-orient

Index chronologique : époque contemporaine, XXe siècle, XXIe siècle

Index géographique : Bulgarie, États-Unis

\section{AUTEUR}

\section{MARIE VRINAT-NIKOLOV}

Professeur des universités - langue et littérature bulgares, Institut NAtional des Langues et Civilisations Orientales (INALCO, Paris) ; Directrice du Centre d'étude de l'Europe médiane (CEEM) ; Co-directrice du Centre de recherches Europes-Eurasie (CREE) ; Traductrice littéraire. 